\title{
ON THE ERGODIC THEOREM FOR POSITIVE OPERATORS ${ }^{1}$
}

\author{
BY LOUIS SUCHESTON
}

Communicated by M. Loève, December 22, 1966

Let $(X, \propto, \mu)$ be a $\sigma$-finite measure space and let $T$ be a positive linear operator on $L_{1}(X, \propto, \mu)$. The ratio ergodic theorem of ChaconOrnstein (see [3], [7], [2]) assumes that $|T|_{1}$, the $L_{1}$ norm of $T$, is less than or equal to one. Here we discuss the behavior of the ratio under the weaker boundedness assumption $\left(b_{h}\right)$. All sets and functions introduced below are assumed measurable. All relations are assumed to hold modulo sets of $\mu$-measure zero. $L_{1}^{+}$is the class of nonnegative not identically vanishing elements of $L_{1}$; similar conventions apply to other function spaces. $L_{1}(A)$ is the class of functions $f$ with supp $f$ (support of $f$ ), contained in $A$ and $\int|f|<\infty . T^{P} g$ is the function $g+T g+T^{2} g+\cdots$. The function $f \cdot 1_{A}$ is sometimes written $f_{A}$. A set $A$ is called closed on a set $B$ if $f \in L_{1}^{+}(A)$ implies $1_{B} \cdot T f$ $\in L_{1}(A)$. A set closed on $X$ is called closed.

THEOREM 1. Let $h$ be a fixed function in $L_{\infty}^{+}$and assume that $T$ satisfies the following condition:

$$
\sup _{n} \int T^{n} f \cdot h<\infty \quad \text { for each } f \in L_{1}^{+} .
$$

Then the space $X$ uniquely decomposes into sets $Y^{h}$ and $Z^{h}$ with the following properties. The set $Z^{h}$ is closed and, if $f \in L_{1}\left(Z^{h}\right)$, then

$$
\gamma^{*}(f) \stackrel{\text { def }}{=} \lim _{n}\left(\sup _{j} n^{-1} \sum_{i=0}^{n-1} \int\left(T^{i+j}|f| \cdot h\right)=0 .\right.
$$

If $f \in L_{1}^{+}\left(Y^{h}\right)$, then $\gamma^{*}(f)>0$.

Theorem 2. Assume $\left(\mathrm{b}_{\mathrm{h}}\right)$. The set $Y^{h}$ decomposes into the conservative part $Y C^{h}$ and the dissipative part $Y D^{h}$ : for each $f \in L_{1}^{+}, T^{P} f=0$ or $\infty$ on $Y C^{h} ; T^{p} f<\infty$ on $Y D^{h}$. The subsets of $Y C^{h}$ closed on $Y^{h}$ form a $\sigma$-field, say $\mathfrak{C}^{h}$, and $Y C^{h} \in \mathfrak{C}^{h}$. If $X \neq Z^{h}$, then the equation

$$
e \in L_{\infty}^{+}, \quad \operatorname{supp} e=Y^{h}, \quad T^{*} e=e
$$

${ }^{1}$ Research supported in part by the National Science Foundation Grant GP-1458. Complete proofs, and some examples will appear in Z. Wahrscheinlichkeitstheorie. 
admits a solution e which on $Y C^{\text {h }}$ is uniquely determined, modulo multiplication by $a \mathfrak{e}^{h}$ measurable function. If

$$
e \cdot f+f_{z} \in L_{1}^{+}, \quad e \cdot g+g_{z} \in L_{\mathbf{1}}^{+},
$$

then the ratio

$$
D_{n}(f, g) \stackrel{\text { dof }}{=} \sum_{i=0}^{n-1} T^{i f} / \sum_{i=0}^{n-1} T^{i} g
$$

converges to a finite limit on the set $Y \cap$ supp $T^{P} g$. The limit is $T^{P} f / T^{P} g$ on $Y D^{h} \cap \operatorname{supp} T^{P} g$ and

$$
\frac{E\left[R\left(T, Y C^{h}, Y D^{h}\right) f \cdot e / \mathbb{e}^{h}\right]}{E\left[R\left(T, Y C^{h}, Y D^{h}\right) g \cdot e / \mathbb{e}^{h}\right]}
$$

on $Y C^{h} \cap \operatorname{supp} T^{P} g$, where

(5) $\quad R(T, A, B) f=f_{A}+\left(T f_{B}\right)_{A}+\cdots+\left(T\left(T^{n} f_{B}\right)_{B}\right)_{A}+\cdots$

(The conditional expectations in (4) are considered as computed with respect to a finite equivalent measure.)

The standing assumption from now on is $\left(\mathrm{b}_{1}\right):\left(\mathrm{b}_{\mathrm{h}}\right)$ with $h=1$, which by the uniform boundedness principle may be stated as:

$$
\sup _{n}\left|T^{n}\right|_{1}<\infty .
$$

The superscript $h=1$ is omitted: we write $Z$ for $Z^{1}$, e for $\mathfrak{C}^{1}$, etc. A stronger statement than Theorem 1 is now true.

Theorem 3. If $f \in L_{1}(Z)$ then $\lim \int T^{n} f=0$; if $f \in L_{1}^{+}(Y)$ then $\lim \inf \int T^{n} f>0$.

Theorem 4. Assume that $X=Y C$ and that the $\sigma$-field $\mathfrak{e}$ is trivial. Then there is a unique, up to multiplicative constants, function e satisfying:

$$
e \in L_{\infty}^{+}, \quad \operatorname{supp} e=X, \quad T^{*} e=e .
$$

If $f \cdot e \in L_{1}^{+}, g \cdot e \in L_{1}^{+}$, then the limit of $D_{n}(f, g)$ is on $X$ :

$$
\frac{\int f \cdot e}{\int g \cdot e}=\lim _{n} \frac{\sup _{j} \sum_{i=0}^{n-1} \int T^{i+j f}}{\sup _{j} \sum_{i=0}^{n-1} \int T^{i+j} g}=\lim _{n} \frac{\inf _{j} \sum_{i=0}^{n-1} \int T^{i+j} f}{\inf _{j} \sum_{i=0}^{n-1} \int T^{i+j} g} .
$$


Theorem 5. Let $g \in L_{1}^{+}(Z)$ and $p \in L_{\infty}^{+}(Z)$ be such that

$$
\sum_{i=0}^{\infty} \int T^{i} g \cdot p=\infty
$$

Then there is a function $f \in L_{1}^{+}$such that

$$
\underset{n}{\lim \sup }\left(\sum_{i=0}^{n-1} \int T^{i} f \cdot p\right) /\left(\sum_{i=0}^{n-1} \int T^{i} g \cdot p\right)=\infty .
$$

Theorem 6. Let $g \in L_{1}^{+}(Z)$ be such that $T^{P} g=\infty$ on $Z$. Then for each function $p \in L_{1}^{+}$there is a function $f \in L_{1}^{+}$with

$$
\lim \sup _{n} \int D_{n}(f, g) \cdot p=\infty \text {. }
$$

We now wish to make a statement about the behavior of the ratio at a point, and this motivates the following definition. The operator $T$ is called asymptotically regular (regular) at a point $x_{0}$ if for all $n$ sufficiently large (for all positive $n$ ), the value of $T^{n} f$ at $x_{0}$ does not depend upon the choice of $f$ in its $L_{1}$ equivalence class. In the discrete case $T$ is regular at each point; more generally, operators regular at each point may be defined by transition measures. By a transition measure we understand a function $T(x, A)$ of two variables which for each fixed $A \in Q$ is a measurable function in $x \in X$; for each fixed $x \in X$, a $\sigma$-finite $\mu$-continuous measure in $A \in Q$. A transition measure $T($,$) acts on L_{1}$ by the relation

$$
T f(x)=\int_{X} T(x, d y) f(y) \quad f \in L_{1} .
$$

THEOREM 7. Let $T$ be asymptotically regular at a point $x_{0} \in Z$ and let $g \in L_{1}^{+}(Z)$ be such that $T^{P} g=\infty$ on $Z$. Then there is a function $f \in L_{1}^{+}$ such that

$$
\limsup D_{n}(f, g)\left(x_{0}\right)=\infty
$$

The following Theorem 8 is concerned with mean convergence to zero. The case $|T|_{1}=1$ has been independently obtained by Krengel and Neveu (see [6]); it is implied by, and implies (cf. [5, p. 662]) an $L_{1}$ decomposition theorem due to Chacon [1]. Mrs. Dowker [4] proved Theorem 8 in the case when $T$ is an isometry of $L_{1}$ of a probability space, generated by a point transformation $\tau$ by the relation $f \circ \tau=T^{*} f, f \in L_{\infty}$. 
THEOREM 8. Let $e \in L_{\infty}^{+}$be such that $\operatorname{supp} e=Y$ and $T^{*} e=e$. If $f \in L_{1}(Y C)$ and $E(f \cdot e / \mathcal{C})=0$, then $n^{-1}\left(f+T f+\cdots+T^{n-1} f\right)$ converges to zero in $L_{1}$ mean.

We now sketch the proof of the main assertions of Theorem 1 and Theorem 2. Let $\left\{L_{\beta}, \beta \in B\right\}$ be the collection of all Banach limits. Assume $\left(b_{h}\right)$. For a fixed $\beta \in B$ we show that there is a $T^{*}$ invariant function $e_{\beta}$, either null or in $L_{\infty}^{+}$, and such that for each $f \in L_{1}$

$$
L_{\beta}\left(\int T^{n} f \cdot h\right)=\int f \cdot e_{\beta} .
$$

Define $Y^{h}$ as the maximal among the sets supp $e_{\beta}, \beta \in \mathrm{B}$. Theorem 1 now follows because the functional applied in (1) to the sequence $\int T^{n}|f| \cdot h$ is the maximal value of Banach limits. Among the functions $e_{\beta}, \beta \in \mathrm{B}$ there is at least one, say $e$, which is a solution of (2). The operator $V$ defined by

$$
V f=e \cdot T\left[f /\left(e+1_{z_{h}}\right)\right]
$$

has the $L_{1}$ norm less than or equal to one, and the convergence of (3) to a finite limit follows by application to this operator of the Chacon-Ornstein theorem.

In conclusion the author wishes to acknowledge the helpful comments of Mr. L. A. Klimko.

\section{BIBLIOGRAPHY}

1. R. V. Chacon, Resolution of positive operators, Bull. Amer. Math. Soc. 68 (1962), $572-574$.

2. - Identification of the limit of operator averages, J. Math. Mech. 11 (1962), 957-961.

3. R. V. Chacon and D. S. Ornstein, $A$ general ergodic theorem, Illinois J. Math. 4 (1960), 153-160.

4. Y. N. Dowker, On measurable transformations in finite measure spaces, Ann. of Math. 62 (1955), 504-516.

5. N. Dunford and J. T. Schwartz, Linear operators. I, Interscience, New York, 1958.

6. U. Krengel, On the global limit behaviour of Markov chains and of general nonsingular Markov processes, Z. Wahrscheinlichkeitstheorie (to appear).

7. J. Neveu, Mathematical foundations of the calculus of probability, Holden-Day, San Francisco, 1965.

The Ohio State University 